\title{
In-flight optical performance assessment for the Metis solar coronagraph
}

\section{Vania Da Deppo, Paolo Chioetto, Vincenzo Andretta, Chiara Casini, Fabio Frassetto, et al.}

Vania Da Deppo, Paolo Chioetto, Vincenzo Andretta, Chiara Casini, Fabio Frassetto, Alessandra Slemer, Paola Zuppella, Marco Romoli, Silvano Fineschi, Petr Heinzel, Giampiero Naletto, Gianalfredo Nicolini, Daniele Spadaro, Marco Stangalini, Luca Teriaca, Alessandro Bemporad, Marta Casti, Michele Fabi, Catia Grimani, Klaus Heerlein, Giovanna Jerse, Federico Landini, Alessandro Liberatore, Enrico Magli, Radek Melich, Maurizio Pancrazzi, Maria-G. Pelizzo, Paolo Romano, Clementina Sasso, Thomas Straus, Roberto Susino, Michela Uslenghi, Cosimo Antonio Volpicelli, "In-flight optical performance assessment for the Metis solar coronagraph," Proc. SPIE 11852, International Conference on Space Optics — ICSO 2020, 1185210 (11 June 2021); doi: 10.1117/12.2599220

SPIE Event: International Conference on Space Optics - ICSO 2021, 2021, Online Only 


\section{International Conference on Space Optics-ICSO 2020}

Virtual Conference

30 March-2 April 2021

Edited by Bruno Cugny, Zoran Sodnik, and Nikos Karafolas
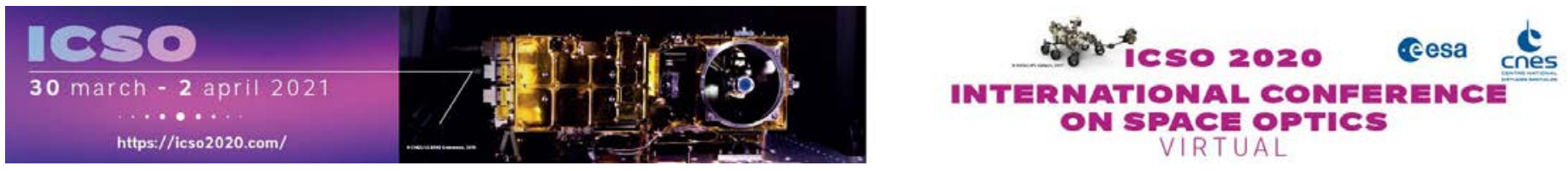

\section{In-flight optical performance assessment for the Metis solar coronagraph}

\section{Cesa isoporecedings denes}




\title{
In-flight optical performance assessment for the Metis solar coronagraph
}

\begin{abstract}
Vania Da Deppo ${ }^{* a, b}$, Paolo Chioetto ${ }^{\mathrm{a}, \mathrm{b}, \mathrm{c}}$, Vincenzo Andretta ${ }^{\mathrm{d}}$, Chiara Casini $^{\mathrm{a}, \mathrm{b}, \mathrm{c}}$, Fabio Frassetto ${ }^{\mathrm{a}, \mathrm{e}}$, Alessandra Slemer ${ }^{\mathrm{a}, \mathrm{b}}$, Paola Zuppella ${ }^{\mathrm{a}, \mathrm{b}}$, Marco Romolif ${ }^{\mathrm{f}}$, Silvano Fineschi ${ }^{\mathrm{e}}$, Petr Heinzel ${ }^{\mathrm{g}}$, Giampiero Naletto $^{\mathrm{h}, \mathrm{a}, \mathrm{b}, \mathrm{c}}$, Gianalfredo Nicolini ${ }^{\mathrm{e}}$, Daniele Spadaro ${ }^{\mathrm{i}}$, Marco Stangalini ${ }^{\mathrm{j}}$, Luca Teriaca ${ }^{\mathrm{k}}$, Alessandro

Bemporad $^{\mathrm{e}}$, Marta Casti ${ }^{1}$, Yara De Leo ${ }^{\mathrm{k}, \mathrm{m}}$, Michele Fabi ${ }^{\mathrm{n}, \mathrm{o}}$, Catia Grimani ${ }^{\mathrm{n}, \mathrm{o}}$, Klaus Heerlein ${ }^{\mathrm{k}}$,

Giovanna Jerse $^{\mathrm{p}}$, Federico Landini ${ }^{\mathrm{e}}$, Alessandro Liberatore ${ }^{\mathrm{e}}$, Enrico Magli ${ }^{\mathrm{q}}$, Radek Melich ${ }^{\mathrm{r}}$,

Maurizio Pancrazzi ${ }^{\mathrm{e}}$, Maria-G. Pelizzo ${ }^{\mathrm{a}}$, Paolo Romano ${ }^{\mathrm{i}}$, Clementina Sasso ${ }^{\mathrm{d}}$, Thomas Straus ${ }^{\mathrm{d}}$, Roberto Susino ${ }^{\mathrm{e}}$, Michela Uslenghi ${ }^{\mathrm{s}}$, Cosimo Antonio Volpicelli ${ }^{\mathrm{e}}$

${ }^{a}$ CNR-Istituto di Fotonica e Nanotecnologie, Padova, Via Trasea 7, 35131 Padova, Italy; ${ }^{\text {b INAF- }}$

Osservatorio Astronomico di Padova, Vicolo dell' Osservatorio 5, 35122 Padova, Italy; ${ }^{\circ} \mathrm{CISAS}$

Centro Interdipartimentale Studi e Attività Spaziali “G. Colombo", Via Venezia 15, 35131 Padova,

Italy; ${ }^{\mathrm{d}}$ INAF-Osservatorio Astronomico di Capodimonte, Salita Moiariello 16, 80131 Napoli, Italy;

INAF-Osservatorio Astrofisico di Torino, Strada Osservatorio 20, 10025 Pino Torinese (To), Italy;

fDipartimento di Fisica ed Astronomia - Università degli Studi di Firenze, Via G. Sansone 1, 50019

Sesto Fiorentino (Fi), Italy; ${ }^{g}$ Academy of Science of the Czech Republic, Narodni 3, 11720 Prague

1, Czech Republic; 'Dipartimento di Fisica e Astronomia "Galileo Galilei” - Università degli Studi

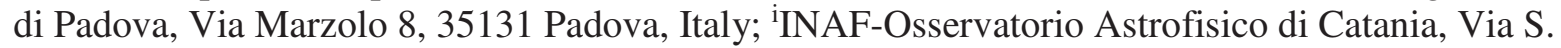

Sofia 78, 95123 Catania, Italy; ${ }^{\mathrm{j} A S I}$, Via del Politecnico, 00133 Roma, Italy; ${ }^{\mathrm{k}}$ Max Planck Institute

for Solar System Research, Justus-von-Liebig-Weg 3, 37077 Göttingen, Germany; ${ }^{1}$ Catholic

University @ NASA-GSFC, Maryland, USA; mDipartimento di Fisica ed Astronomia "Ettore

Majorana"- Sezione Astrofisica, Università degli Studi di Catania, Via S. Sofia 78, 95123 Catania,

Italy; ${ }^{n}$ Università degli Studi di Urbino Carlo Bo, Via S. Chiara 27, 61029 Urbino (PU), Italy;

${ }^{\circ}$ INFN Sezione di Firenze, Via G. Sansone 1, 50019 Sesto Fiorentino (Firenze), Italy; ${ }^{\text {INAF- }}$

Osservatorio Astronomico di Trieste, Via Giambattista Tiepolo 11, 34143 Trieste, Italy; ${ }^{9}$ Politecnico

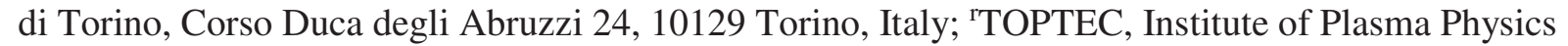

ASCR, Sobotecká 1660, 51101 Turnov, Czech Republic; 'sINAF-IASF, Via Alfonso Corti 12,

20133 Milano, Italy
\end{abstract}

\begin{abstract}
Metis is a multi-wavelength coronagraph onboard the European Space Agency (ESA) Solar Orbiter mission. The instrument features an innovative instrument design conceived for simultaneously imaging the Sun's corona in the visible and ultraviolet range. The Metis visible channel employs broad-band, polarized imaging of the visible K-corona, while the UV one uses narrow-band imaging at the HI Ly $\alpha$, i.e. $121.6 \mathrm{~nm}$.

During the commissioning different acquisitions and activities, performed with both the Metis channels, have been carried out with the aim to check the functioning and the performance of the instrument. In particular, specific observations of stars have been devised to assess the optical alignment of the telescope and to derive the instrument optical parameters such as focal length, PSF and possibly check the optical distortion and the vignetting function.

In this paper, the preliminary results obtained for the PSF of both channels and the determination of the scale for the visible channel will be described and discussed. The in-flight obtained data will be compared to those obtained on-ground during the calibration campaign.
\end{abstract}

Keywords: Metis coronagraph, Solar Orbiter mission, optical performance, PSF

*vania.dadeppo@ifn.cnr.it; phone +39-0499815639 


\section{INTRODUCTION}

Solar Orbiter (SO) is a mission dedicated to study solar and heliospheric physics[1]. It was selected as the first mediumclass mission of ESA's Cosmic Vision 2015-2025 Programme. With a combination of in-situ and remote sensing instruments, $\mathrm{SO}$ is conceived for the circumsolar region exploration with the purpose of giving an answer to the scientific questions on how the heliosphere is generated and controlled by the Sun.

Launched in February 2020, the mission will provide close-up, high-latitude observations of the Sun. SO will have a highly elliptic orbit ( $0.9 \mathrm{AU}$ at aphelion and $0.28 \mathrm{AU}$ at perihelion). Thanks to its mission profile, for the first time the poles of the Sun and the circumsolar region will be seen and studied from a privileged point of view near the Sun.

Soon after launch SO underwent the Near Earth Commissioning Phase (NECP), the spacecraft and the payload were functionally checked and calibrated. Also the Metis coronagraph [2], which is one of the remote sensing instruments mounted on-board the SO spacecraft, was switched on, functionally tested and characterized [3]. In particular, the two mechanisms of the instrument, the one-shot sealing cap and the internal occulter mechanism (IOM), were activated.

Metis has been conceived to acquire both visible and UV images of the solar corona. It has two channels: one to study the UV HI Lyman $\alpha$ at $121.6 \mathrm{~nm}$, and the other for the polarized visible light band $(580-640 \mathrm{~nm})$.

Metis adopts a configuration called 'inverted externally occulted' that is able to prevent the solar disk light to enter the telescope; this light is back reflected outside by a mirror (M0) [4]. The passing coronal light is collected by the Metis telescope. Common to both channels, the Gregorian on-axis telescope is centrally occulted and both the primary and the secondary mirrors have annular shape. The on-axis field is not available since it is anyway completely blocked by the M0 mirror; for the alignment and test on-ground an ad hoc alignment set-up has been developed for the telescope alignment [5].

In this paper, after a description of the Metis optical design, the preliminary measured telescope performance in-flight will be presented and compared to the one obtained during the on-ground calibration campaign [6].

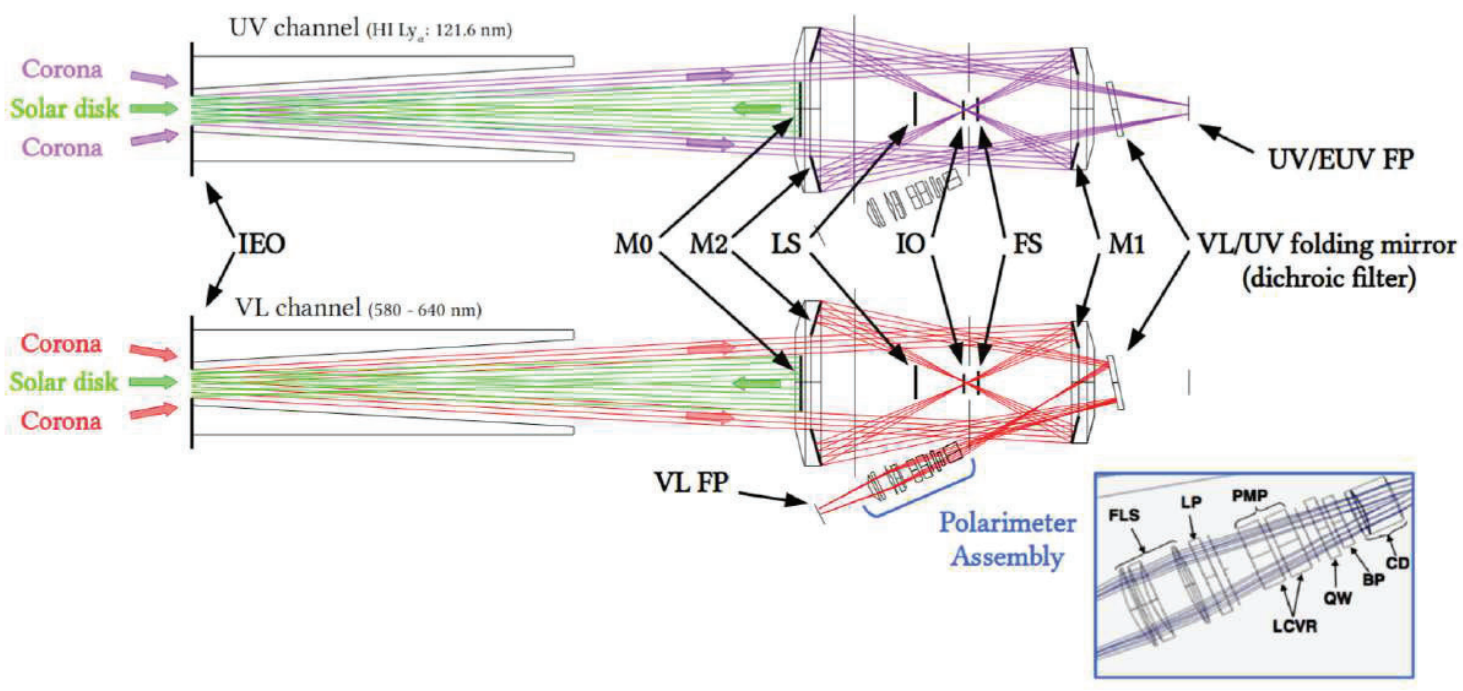

Figure 1. Metis layout. The raytraces of the two channels are depicted separately. On the top the UV channel and on the bottom the VL one with a zoom on the VL polarimeter assembly.

\section{METIS OPTICAL DESIGN AND PERFORMANCE}

\subsection{Metis optical design}

The Metis instrument is conceived to image the solar corona from a near-Sun orbit in two different spectral bands: the UV narrow band HI Lyman- $\alpha$ at $121.6 \mathrm{~nm}$, and the polarized broad-band visible light $(580-640 \mathrm{~nm})$. The instrument is 
designed to image the structure and dynamics of the full corona in the range from 1.6 to 3.1 solar radii $\left(\mathrm{R}_{\mathrm{s}}\right)$, at minimum perihelion distance $(0.28 \mathrm{AU})$, and from $2.8 \mathrm{R}_{\mathrm{s}}$ to $5.5 \mathrm{R}_{\mathrm{s}}$, at $0.5 \mathrm{AU}$. The annular Field of View (FoV) covered by the instrument is between $1.6^{\circ}$ and $2.9^{\circ}$, and the attained spatial resolution is $20^{\prime \prime}$.

The "inverted externally occulted" configuration (see Figure 1) [7][8] allows to reduce the thermal loads inside the instrument. The inverted external occulter (IEO), a small aperture on the spacecraft thermal shield, also acts as the entrance pupil of the instrument. The solar disk light passing through the IEO is back-reflected by a spherical heat-rejection mirror (M0) and focused on the IEO itself. The coronal light, on the other hand, is collected by an on-axis Gregorian telescope.

The primary mirror (M1) focuses the gathered coronal light in its focal plane, where a field stop (FS) is placed and limits the outer FOV of the coronagraph. Then the secondary mirror (M2) focalizes the light again; part of the light passes through the interference filter (IF) and then is collected by UV detector; the other part is reflected by the IF and is directed to the polarimeter assembly.

Stray light minimization is a mandatory goal in any coronagraph and the worst offenders are the surfaces hit by the direct solar disk light, IEO and M0. The internal occulter (IO) and the Lyot stop (LS) are, respectively, conjugated with the IEO and M0 via the primary mirror M1. IO blocks the solar disk light diffracted by the IEO edge, while LS blocks the light diffracted by the M0 edge [9][10].

Metis consists of a single optical head that incorporates 2 different channels: a UV imaging channel and a polarimeter visible light (VL) channel. The telescope optics (M1 and M2) and the stop system (IEO, M0, FS, IO and LS) are common to both channels, while the VL channel has a dedicated relay optics and a polarizing group. Each channel has its own detector respectively called UVDA and VLDA.

The Metis instrument optical specifications are summarized in Table 1.

Table 1 Summary of Metis instrument optical characteristics.

\begin{tabular}{|ll|}
\hline \multicolumn{2}{|c|}{ Metis instrument optical specifications } \\
FoV & $1.6^{\circ}-2.9^{\circ}$ \\
& $1.7-3.1 \mathrm{Rs} @ 0.28 \mathrm{AU}-3.0-5.5 \mathrm{R}_{\mathrm{s}} @ 0.5 \mathrm{AU}$ \\
Telescope type & Externally occulted on-axis Gregorian \\
Effective focal length & $\mathrm{VL}: 200 \mathrm{~mm}$ \\
& $\mathrm{UV}: 300 \mathrm{~mm}$ \\
Wavelength range & $\mathrm{VL}: 580-640 \mathrm{~nm}$ \\
& $\mathrm{UV}: 121.6 \pm 10 \mathrm{~nm}$ \\
& $\mathrm{VL}:<20 \operatorname{arcsec} @>2^{\circ}$ \\
Spatial resolution & $<40 \operatorname{arcsec} @<2^{\circ}$ \\
& $\mathrm{UV}:<20 \operatorname{arcsec}$ \\
Average Straylight & $\mathrm{VL}<10^{-9}$ \\
(B $\left.{ }_{\text {cor }} / \mathrm{B}_{\text {sun }}\right)$ & $\mathrm{UV}<10^{-7}$ \\
Detectors & $\mathrm{VL}: 2048 \times 204810 \mu \mathrm{m}$ pixel size \\
\end{tabular}

\subsection{Estimated UV and Visible-light imaging path optical performance}

The coronal light collected by the Metis Gregorian on-axis telescope is divided in two different paths:

- $\quad$ narrow band ultraviolet HI Lyman $\alpha(121.6 \mathrm{~nm})$ path, corresponding to the picture on the top in Figure 1;

- $\quad$ polarized visible-light $(580-640 \mathrm{~nm})$ path, corresponding to the picture on the bottom in Figure 1.

The UV light detector assembly (UVDA) consists of three main elements: (a) an intensifier with $\mathrm{KBr}$ photocathode in optical contact (via a phosphor screen) with (b) a fiber optics 2:1 de-magnifying coupler connected to (c) a CMOS APS STAR1000 (1k x 1k format, $15 \mu \mathrm{m}$ square pixel). So the equivalent pixel size at the focal plane for the UV channel is 30 $\mu \mathrm{m}$. The visible light detector assembly (VLDA) consists of a visible light camera equipped with a CMOS active pixel sensor (APS), $2 \mathrm{k}$ x 2k, $10 \mu \mathrm{m}$ pixel [2]. 
The two different UV and VL paths are separated through a UV interference filter (IF), inserted in the converging beam exiting M2, and inclined at $12^{\circ}$ with respect to the optical axis of the telescope. The UV narrow bandpass interference filter acts as VL-UV dichroic beam splitter by selecting the $121.6 \mathrm{~nm} \mathrm{UV}$ band in transmission and reflecting the VL.

The mirrors are coated with $\mathrm{Al}+\mathrm{MgF}_{2}$ with high reflectivity in the $\mathrm{VL}$ band and optimized to have an enhanced reflectivity at $121.6 \mathrm{~nm}[11][12]$.

The VL path aberrations are rather limited and the theoretical performance is diffraction limited. See Figure 2 for the expected VL channel performance in terms of RMS spot radius, both the tangential (tg) and radial (r) RMS have been calculated. Tangential and radial refer to the solar corona.

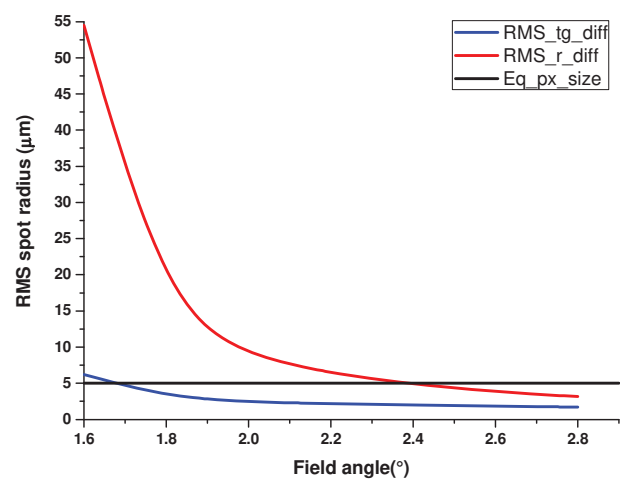

Figure 2. VL theoretical diffraction RMS spot size.

The UV path performance is not radially symmetric, the IF is a thick $6 \mathrm{~mm}$ tilted plate placed in a converging beam, thus some aberrations (mainly astigmatism and coma) are introduced by the filter [13]. To mitigate these aberrations, the IF surfaces are not parallel but tilted by about $0.15^{\circ}$. The IF is made of a birefringent material, another birefringent window is mounted in front of the detector.

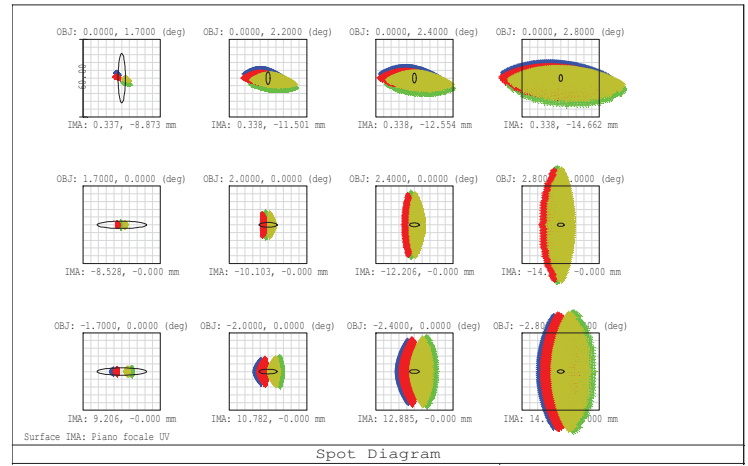

(a)

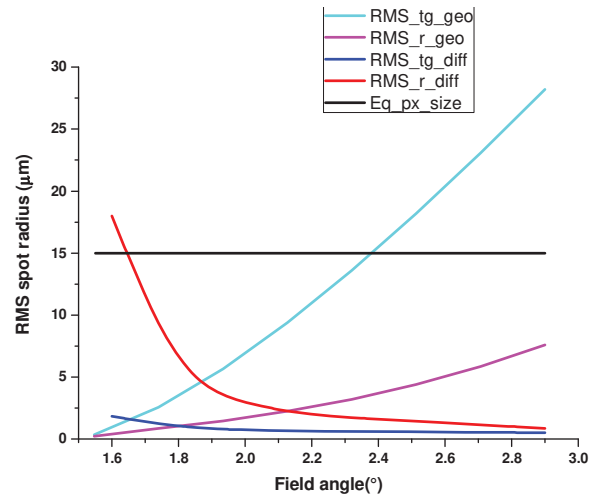

(b)

Figure 3. Metis UV channel performance. (a) Geometric spot diagrams over the FoV both ordinary and extraordinary rays have been depicted; the square boxes have 60 micron size equivalent to $2 \times 2$ pixels of the detector. The Airy disc is also drawn for comparison. (b) Geometrical RMS spot radius versus FoV compared with the diffraction limited RMS spot radius and with the detector equivalent pixel size.

The theoretical optical performance of the UV channel, including an estimation of the diffraction effect, is shown in Figure 3. In Figure 3(a) the spot diagrams over the FoV are shown; the different colors refer to ordinary and extraordinary rays for both the IF and the detector window. In Figure 3(b) the geometrical RMS spot radius calculated at the UV paraxial 
focus for both the tangential and radial direction is compared with the diffraction RMS and with the equivalent pixel size of the UV detector. The UV pixel equivalent size considered is $15 \mu \mathrm{m}$, i.e. half of the UV detector equivalent pixel size.

\subsection{Metis vignetting function}

The light passing through the Metis telescope body is highly vignetted by M0 and then vignetted further by the IO and LS. Moreover, on the diagonals of the image, the two four-spider systems supporting respectively the M0/Lyot Stop assembly and the Internal Occulter/Field Stop assembly are vignetting the beam even more. In Figure 4(a), the whole opto-mechanical layout of Metis is presented and in the bottom right part of the picture the two Internal Occulter/Field Stop and M0/Lyot Stop assemblies with the supporting spiders are shown. The internal occulter mechanism, with the fourspider system clearly visible, is shown in Figure 4 (b).

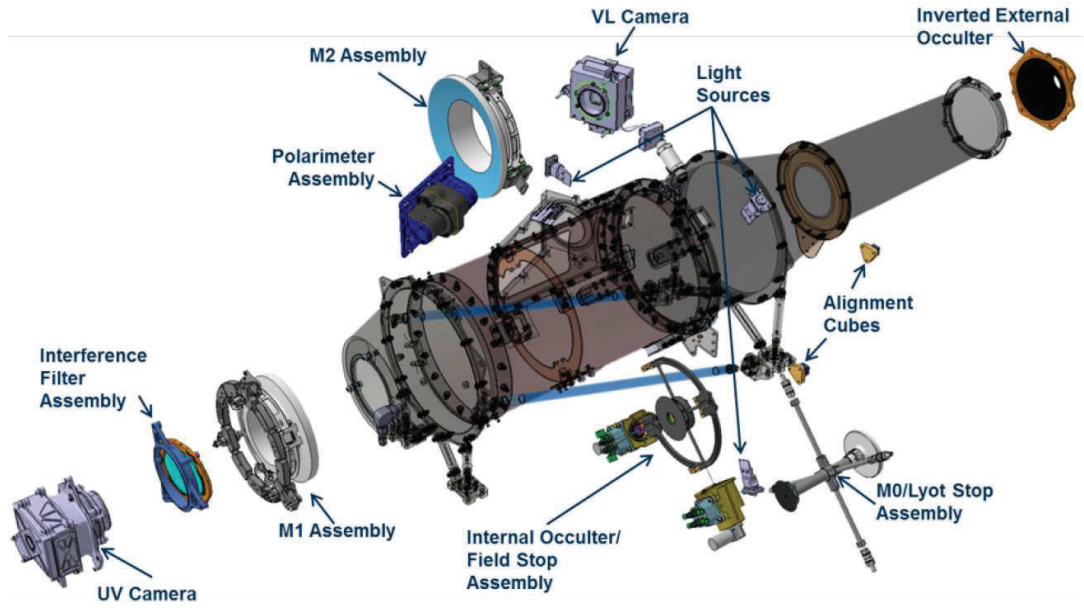

(a)

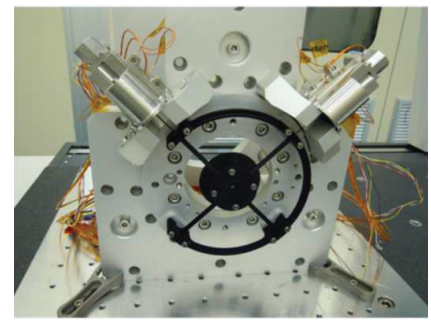

(b)

Figure 4. In (a) opto-mechanical subsystems of Metis. In (b) picture of the flight model of the internal occulter mechanism with the four-spider system painted in black clearly visible [2].

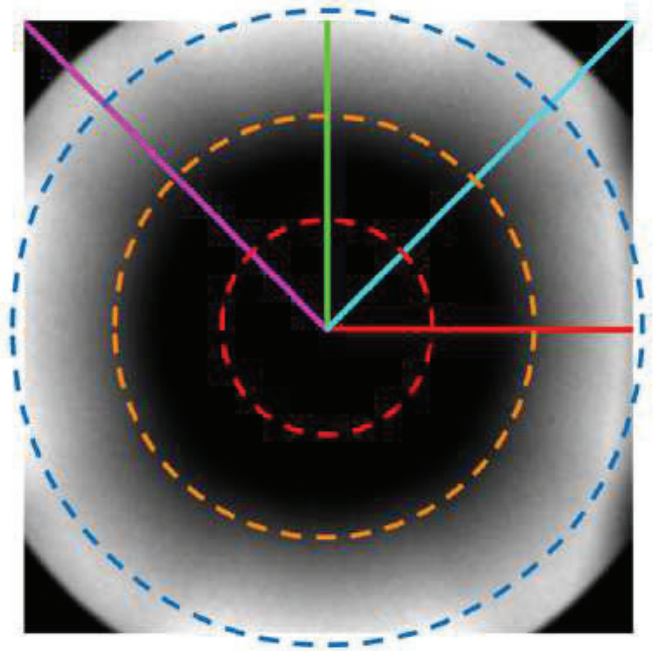

(a)

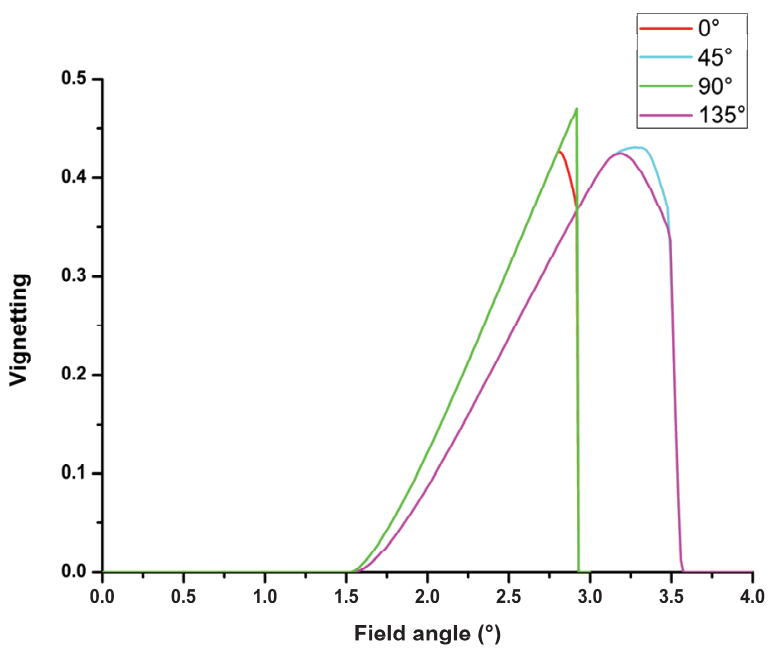

(b)

Figure 5. Dependence of the Metis vignetting function on the radial field of view. (a): Gray scale representation of the bidimensional vignetting function, as obtained by ray-tracing; overlapped dashed circles represent $1^{\circ}$ (red), $2^{\circ}$ (orange), and

$3^{\circ}$ (blue) FoVs. (b): plots of the vignetting function along the radial directions shown in (a) as a function of the different colours. 
Over the nominal FoV of the telescope the fraction of unvignetted rays is always less than 50\%. See Figure 5 for the Metis theoretical vignetting function given both in terms of 2D image in (a) and as radial profiles in (b). The further vignetting due to the spiders is clearly visible by comparing the profiles at $0^{\circ}$ and $90^{\circ}$ with the $45^{\circ}$ and $135^{\circ}$ ones.

\section{IN-FLIGHT OPTICAL PERFORMANCE}

On the 9th of February 2020, the Solar Orbiter spacecraft has been launched by an Atlas V rocket from Cape Canaveral. Soon after launch, the switch on and commissioning of the payload instruments started.

Star observations with Metis are very important to characterize and calibrate the instrument, but at present only some specific sequences have been run specifically for stellar calibration. Anyway in the VL channel stars are commonly seen in the FoV since some are bright enough to stay above the coronal continuum. Some specific UV stars, useful to characterize the UV channel, have also been observed.

\subsection{UV channel performance}

For the UV channel $\alpha$ and $\rho$ Leonis stars have been observed transiting throughout the Metis FoV from east to west approximately at the center of the frame, this direction corresponds to the Y direction of the detector. Images have been acquired using masking and radialization, an example of the images is reported in Figure 6(a).

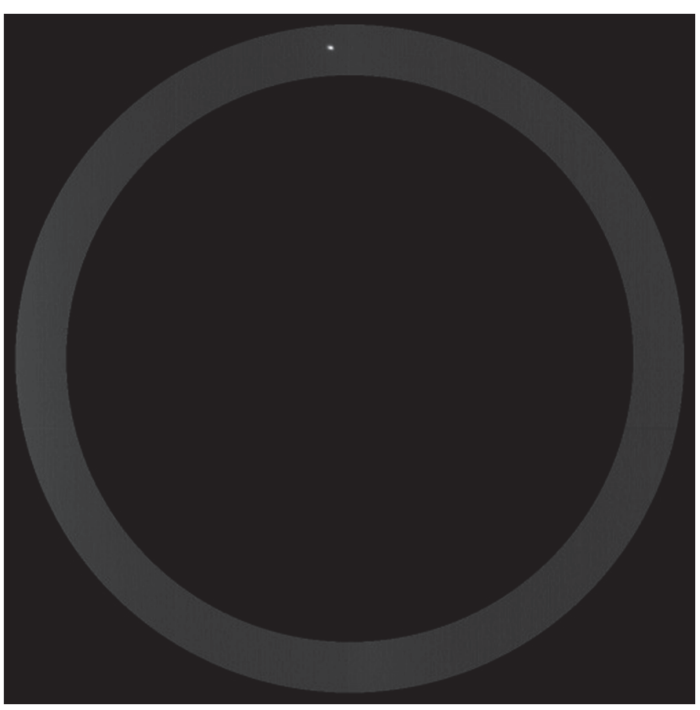

(a)

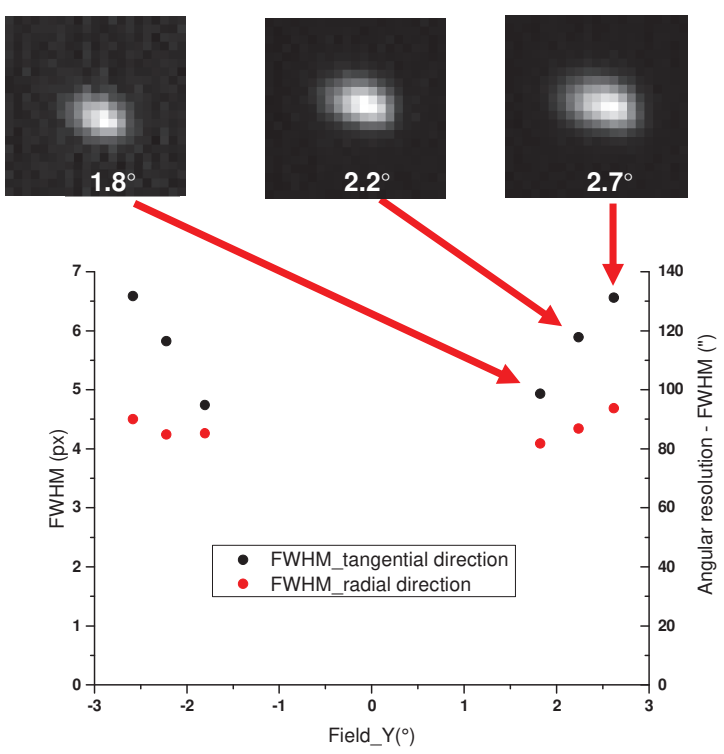

(b)

Figure 6. (a) UV image of $\alpha$ Leo, the bright spot on the top, acquired with masking and radialization. (b) UV PSF measured in-flight using $\alpha$ Leo.

A gaussian fit has been used to estimate the FWHM of the images of the stars and the radial and tangential FWHM have been derived (see Figure 6(b)). Also a comparison with the data obtained on-ground has been done (see Figure 7a and b).

The preliminary estimated UV PSF in-flight is consistent with the one measured on-ground, even if the one measured onground has been measured along the $\mathrm{X}$ axis of the detector, while the one in-flight is measured along the $\mathrm{Y}$ axis. In fact, the spots are elongated in different directions, i.e. in flight they are along the $\mathrm{X}$ detector direction and on-ground along the Y (see Figure 6(b) and Figure 7(a)), nevertheless the PSF radial and tangential FWHM values can be directly compared. 


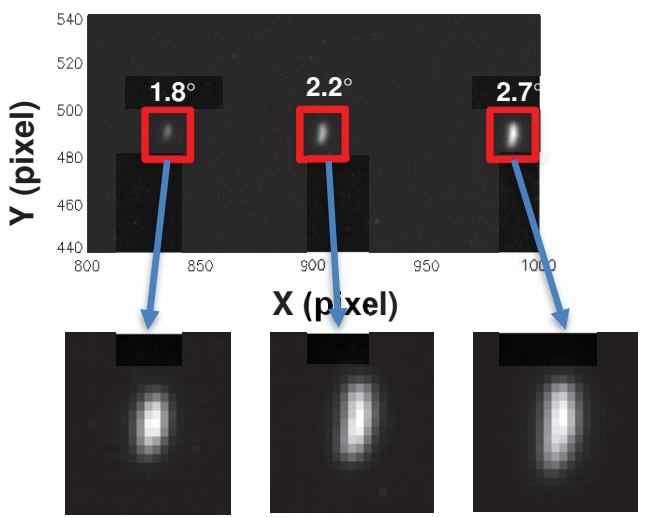

(a)

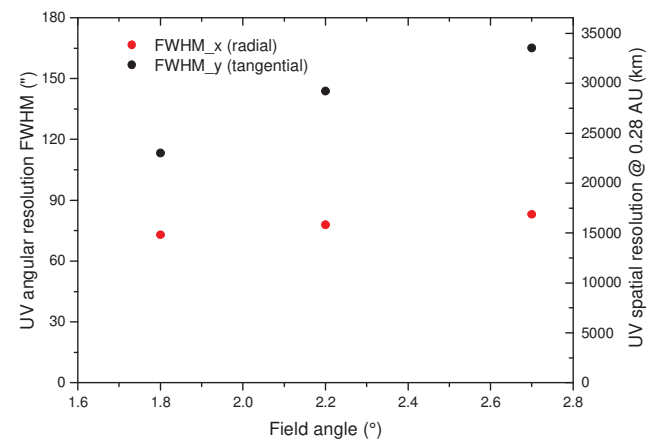

(b)

Figure 7. UV PSF measured on-ground. In (a) appearance of the acquired pinhole images, in (b) measured radial and tangential angular resolution and FWHM of the PSF.

\subsection{VL channel performance}

During the IT-6B1 activity [3], performed in mid-May 2020, some of the brightest stars of the Taurus constellation have been observed. Unfortunately, this sequence had been foreseen with the aim of doing the IO coarse alignment, and so not all of the acquired images are useful and moreover, due to the IO different position from one image to the other, the vignetting function was slightly varying over the different acquired images. Nevertheless, some of the stars have been recognized and their PSFs have been measured.

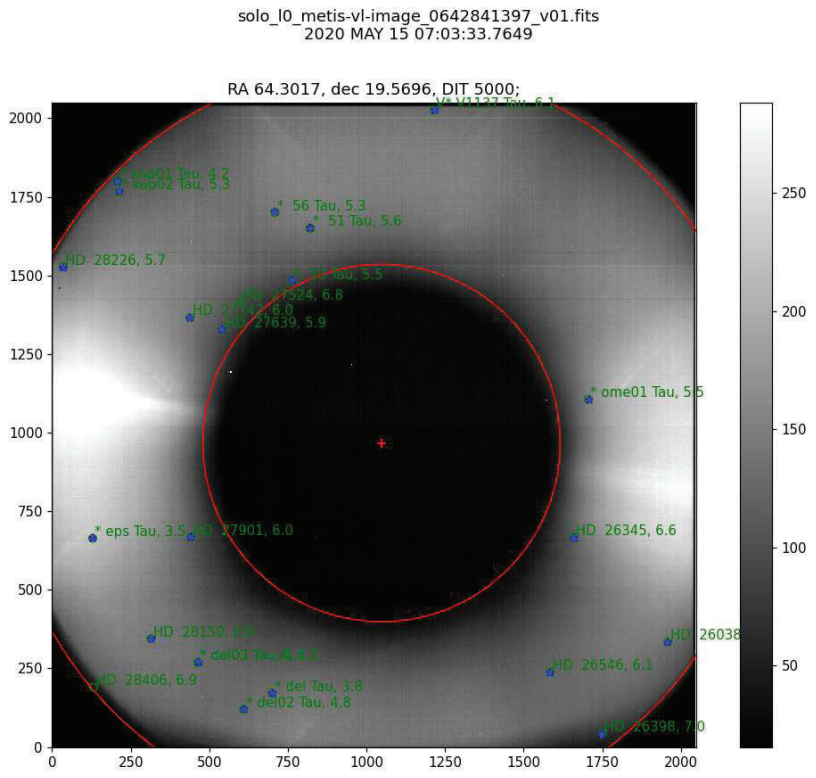

Figure 8 Example of a VL image acquired during the IT-6B1. The identified stars are highlighted and named.

In Figure 8 an example of a VL acquired image is given. Superimposed on the image the detected stars are clearly marked with a blue star symbol and the name and the visual magnitude $\left(\mathrm{m}_{\mathrm{V}}\right)$ of each star are written in green color. This image has been rotated in order to be quasi east-west (EW) oriented along the horizontal direction and south-north (SN) in the vertical direction. The red circles correspond with the inner and outer dimensions of the FoV of Metis, the inner is determined by the vignetting function while the outer by the field stop size. The red cross, almost at the center of the image, corresponds to the boresight. 
In Table 2 a summary of the brightest identified stars is given. The spectral types were obtained from the Simbad database [14]; in parenthesis spectral type from the Bright Star Catalog $5^{\text {th }}$ Revised Edition [15]. The R magnitude is also provided when available. The $\mathrm{R}$ band is centered at $658 \mathrm{~nm}$, and thus it is closer than the $\mathrm{V}$ band to the Metis VL channel wavelength band $(580-640 \mathrm{~nm})$.

Table 2 Summary of brightest stars detected by Metis during the IT-6B1 activity performed 15 May 2020

\begin{tabular}{|llcccc|}
\hline HD & Name & $\begin{array}{c}\text { Sp Type } \\
\text { G9.5IIICN0.5 }\end{array}$ & B-V & mv & $\mathbf{m}_{\mathbf{R}}$ \\
27697 & $\delta_{1}$ Tau & K0-IIICN0.5) & 3.76 & 5.56 \\
27819 & $\delta_{2}$ Tau & A2Vs (A7V) & 0.15 & 4.80 & 4.64 \\
27962 & $\delta_{3}$ Tau & 2IV-Vs (A2IV) & 0.07 & 4.30 & 6.18 \\
27934 & $k_{1}$ Tau & A7IV-V & 0.17 & 4.20 & N/A \\
27946 & $k_{1}$ Tau & F0Vn (A7V) & 0.28 & 5.26 & N/A \\
28305 & $\varepsilon_{1}$ Tau & G9.5IIICN0.5 & 1.01 & 3.53 & N/A \\
26162 & $\omega_{1}$ Tau & K2III & 1.08 & 5.50 & N/A \\
28226 & - & Am & 0.27 & 5.71 & 5.55 \\
26038 & - & K5III & 1.10 & 5.94 & N/A \\
\hline
\end{tabular}

At least 5 of the acquired images have been judged worth detecting the stars and measuring the FWHM of their PSFs.

A composite image has been created, after bias and dark removal, summing some of the different consecutive images acquired. The SO spacecraft is pointing the Sun, so the stars appear to move from one frame to the other approximately along the columns of the detector which are approximately oriented EW with respect to the Sun.

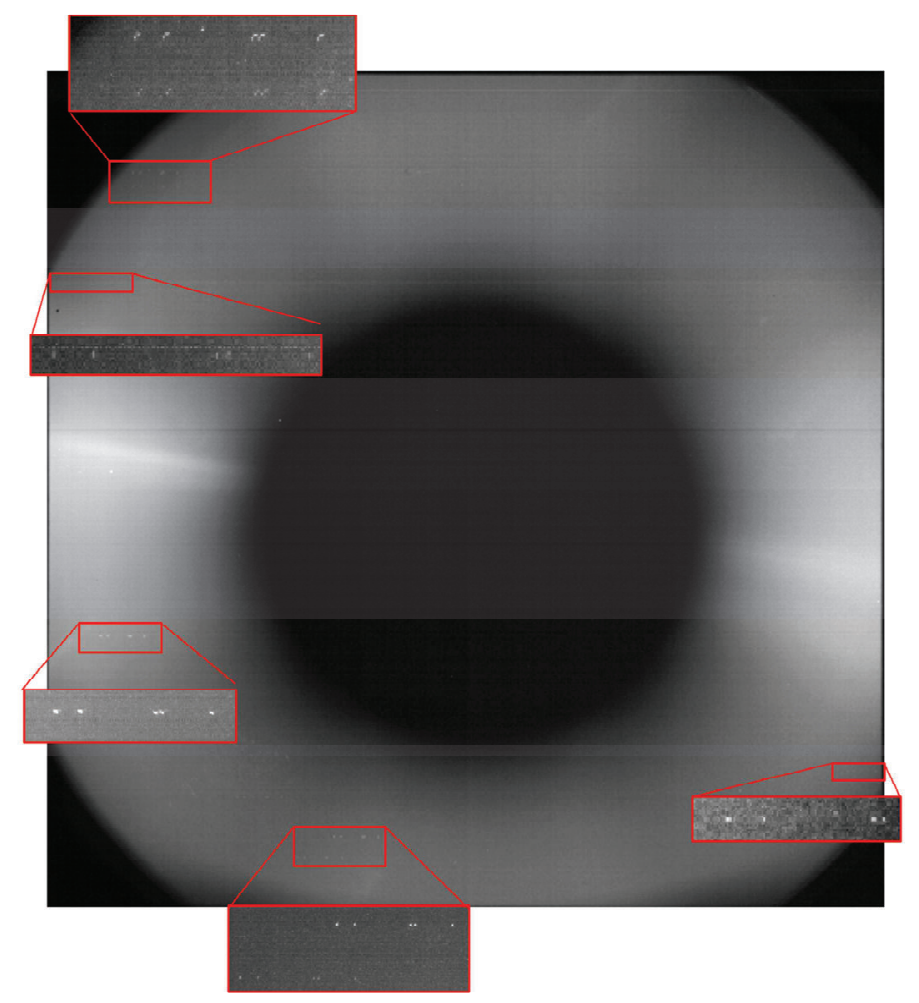

Figure 9. Composite VL image. In the insets, zooms of some of the star images. 
See Figure 9 for the composite image and the appearance of the stars moving on the detector. On the whole image some zoomed insets are depicted to highlight the regions where the stars are visible.

The 5 considered images were acquired respectively 30, 100, 10 and 70 minutes apart one from the other and the stars appear to move from left to right in the frame, i.e. EW.

The VL channel images analyzed so far are of stars not completely well exposed and not uniformly distributed on the detector. There are hints, though to be assessed, that on the edges of the FoV the PSF is larger than expected, particularly in the region where the image vignetting is highly determined by the spiders obscuration as discussed in par. 2.3.

The quite well-exposed stars analyzed, epsilon and delta Tau, suggest that the mean FWHMs, on the left side of the detector, are of the order of 1.5-2 px (see Figure 10(a) and (b)), which are in quite good agreement with the data acquired on-ground (see Figure 11).

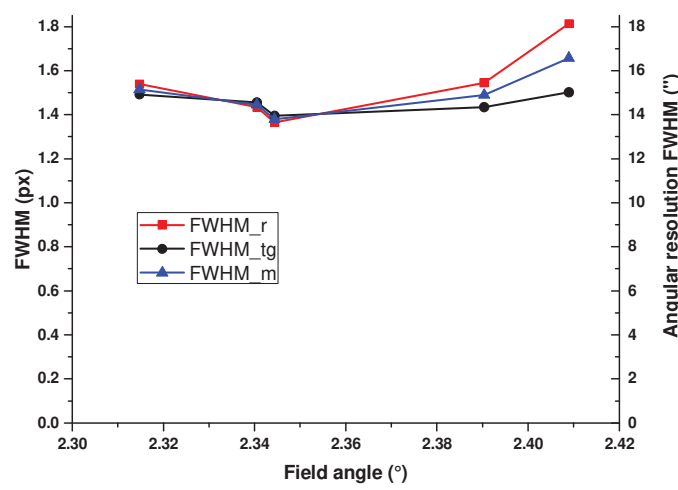

(a)

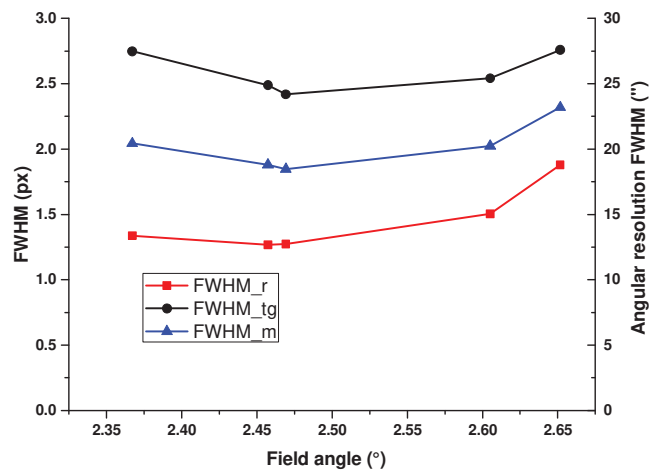

(b)

Figure 10 In (a) PSF FWHM (radial, tangential and mean) measured for $\delta_{1}$ Tau, in (b) for $\varepsilon_{1}$ Tau.

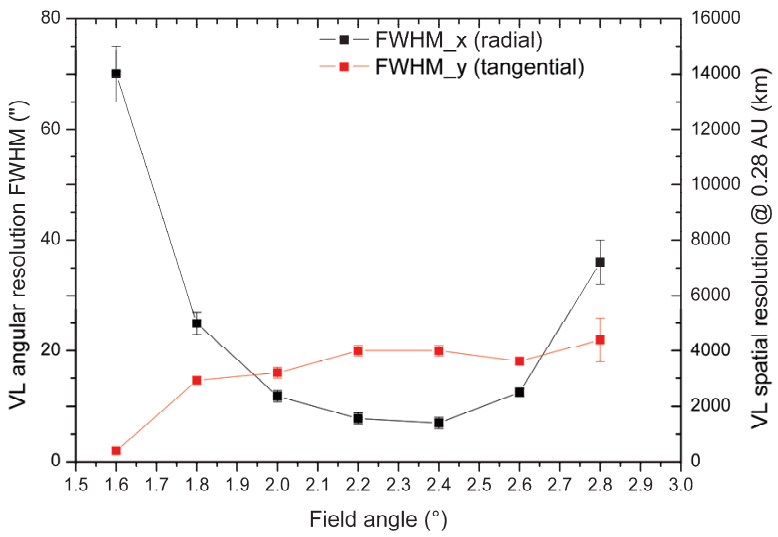

Figure 11 VL channel PSF measured on-ground.

\subsection{Spatial scale}

For the VL channel, having about 10 identified stars in the FoV, an initial estimation of the plate scale and pointing of the spacecraft have been done.

Firstly, the positions of the identified stars on the sensor have been determined using the peak pixel, i.e. the pixel with the highest DN value, of each stellar PSF. 
Then a set of linear transformations has been defined mapping sensor coordinates to celestial coordinates, using the convention detailed in [16], and consisting of a rotation, a translation and a scaling, followed by a gnomonic projection. The corresponding five parameters (the angle of rotation, a pair of translation coordinates and a pair of scaling factors) were then optimized by minimizing the distance on the sensor between the expected position with the measured position of each visible star on the sensor, using a least squares fitting algorithm.

The resulting scaling factors along the $\mathrm{X}$ and $\mathrm{Y}$ direction of the detector were 10.139 and $10.127 " / \mathrm{px}$ respectively, with a standard deviation of $0.005 \% / \mathrm{px}$, which are in agreement with the measurements done on-ground. The measured on-ground focal length for the VL channel after alignment is about $203.5 \mathrm{~mm}$, that is slightly larger than the theoretical $200 \mathrm{~mm}$ one. The measured on-ground focal length corresponds to a pixel scale of about 10.13"/px.

\section{CONCLUSIONS}

Some results on the in-flight optical performance of the Metis coronagraph have been presented. As a preliminary achievement, the PSF FWHMs of the UV channel measured in-flight are perfectly matching those measured on-ground. As for the VL channel, the present available images are not entirely useful to correctly determine the PSF behaviour all over the FoV. Future specific acquisitions will be envisaged to better characterize the system performance; in particular to better sampling the FoV with well exposed stars, to correlate the optical characteristics of one channel with the other one and to verify the relative alignment.

For the VL channel, also a preliminary assessment of the plate scale has been done. The results obtained are in agreement with the measurements done during the on-ground calibration campaign.

\section{ACKNOWLEDGMENTS}

The Metis program is supported by the Italian Space Agency (ASI) under the contracts to the co-financing National Institute of Astrophysics (INAF): Accordi ASI-INAF N. I-043-10-0 and Addendum N. I-013-12-0/1, Accordo ASI-INAF N. 2018-30-HH.0 and under the contracts to the industrial partners: ASI-TASI N. I-037-11-0 and ASI-ATI N. 2013-057I.0. The Metis team thanks Barbara Negri, Enrico Flamini, Marco Castronuovo of the Italian Space Agency and Roberto Della Ceca, Giuseppe Malaguti of the Istituto Nazionale di Astrofisica for their continuous support during the development of the coronagraph. A special thanks to Filippo Marliani of the European Space Agency for his dedication to the program and his high standard of excellence. The testing of Metis was performed by an industrial consortium constituted by OHB Italia S.p.A. (acting as Prime Contractor towards ASI), Thales Alenia Space Italia S.p.A. (Co-Prime Contractor with the specific responsibility of the instrument AIT). ALTEC has provided logistics and technical support for the INAF Optical Payload Systems. The primary and secondary mirrors were provided as a Czech contribution to Metis; the mirror hardware development was possible thanks to the Czech PRODEX Programme. The UVDA assembly was provided as a German contribution to Metis, thanks to the financial support of DLR (grant 50 OT 1201). The VLDA assembly was provided under Contract 2013- 058-I.0 with the Italian Space Agency (ASI). Metis team thanks the former PI, Ester Antonucci, for leading the development of Metis until the final delivery to ESA.

\section{REFERENCES}

[1] Muller, D., St. Cyr, O. C., Zouganelis, I., Gilbert, H. R., Marsden, R., Nieves-Chinchilla, T., Antonucci, E., Auchere, F., Berghmans, D., Horbury, T. S., Howard, R. A., Krucker, S., Maksimovic, M., Owen, C. J., Rochus, P., Rodriguez-Pacheco, J., Romoli, M., Solanki, S. K., Bruno, R., Carlsson, M., Fludra, A., Harra, L., Hassler, D. M., Livi, S., Louarn, P., Peter, H., Sch uhle, U., Teriaca, L., del Toro Iniesta, J. C., Wimmer-Schweingruber, R. F., Marsch, E., Velli, M., De Groof, A., Walsh, A., and Williams, D., "The Solar Orbiter mission. Science overview," 642, A1 (Oct. 2020).

[2] Antonucci, E. et al., "Metis: the Solar Orbiter visible light and ultraviolet coronal imager," A\&A 642, A10 (2020).

[3] Romoli, M., et al., "Challenges during Metis-Solar Orbiter commissioning phase", in International Conference on Space Optics ICSO 2020, Proc. SPIE, this issue (2021).

[4] Fineschi, S. et al., "Optical design of the multi-wavelength imaging coronagraph Metis for the solar orbiter mission," Exp. Astron. 49, 239-263 (2020). 
[5] Da Deppo, V., et al., "Alignment procedure for the Gregorian telescope of the Metis coronagraph for the Solar Orbiter ESA mission," Proc. SPIE 11180, International Conference on Space Optics - ICSO 2018, 1118076 (2019).

[6] Frassetto, F., et al., "Optical performance of the Metis coronagraph on the Solar Orbiter ESA mission," Proc. SPIE 11180, International Conference on Space Optics — ICSO 2018, 111806Y (2019).

[7] Fineschi, S., Antonucci, E., Romoli, M., Bemporad, A., Capobianco, G., Crescenzio, G., Nicolini, G., Massone, G., Telloni, D., Focardi, M., Landini, F., Pancrazzi, M., Poletto, L., Pelizzo, M.-G., Da Deppo, V., Moses, J. D., Andretta, V., Naletto, G., Nicolosi, P., Spadaro, D., Berlicki, A.,Uslenghi, M., Malvezzi, M., Teriaca, L., Abbo, L., Magli, E., "Novel space coronagraphs: METIS, a flexible optical design for multi-wavelength imaging and spectroscopy," Proc. SPIE 8862, 88620G (2013).

[8] Romoli, M., Landini, F., Antonucci, E., Andretta, V., Berlicki, A., Fineschi, S., Moses, J. D., Naletto, G., Nicolosi, P., Nicolini, G., Spadaro, D., Teriaca, L., Baccani, C., Focardi, M., Pancrazzi, M., Pucci, S., Abbo, L., Bemporad, A., Capobianco, G., Massone, G., Telloni, D., Magli, E., Da Deppo, V., Frassetto, F., Pelizzo, M. G., Poletto, L., Uslenghi, M., Vives, S., Malvezzi, M., "METIS: the visible and UV coronagraph for solar orbiter," Proc. SPIE 10563, 105631M (2017).

[9] Landini, F., et al., "Stray light calibration for the Solar Orbiter/Metis solar coronagraph," to be published in this Proc. SPIE volume, (2018).

[10] Sandri, P., et al., "Stray-light analyses of the multielement telescope for imaging and spectroscopy coronagraph Solar orbiter," Opt. Eng. 57(1), 015108 (2018).

[11] Nardello, M., Zuccon, S., Corso, A. J., Zuppella, P., Gerlin, F., Tessarolo, E. and Pelizzo, M. G., "Study of optical materials to be used on Multi Element Telescope for Imaging and Spectroscopy instrument," Journal of Astronomical Telescopes, Instruments, and Systems 1, 024003 (2015).

[12]Zuccon, S., Napolitani, E., Tessarolo, E., Zuppella, P., Corso, A. J., Gerlin, F., Nardello, M., and Pelizzo, M. G., "Effects of helium ion bombardment on metallic gold and iridium thin films," Optical Materials Express 5(1), 176-187 (2015).

[13] Fischer, R. E., Tadic-Galeb, B., and Yoder, P. R., [Optical System Design], McGraw Hill, (2008).

[14] http://simbad.u-strasbg.fr/simbad/

[15] Hoffleit, D. and Warren, Jr., W.H., "The Bright Star Catalog, 5th Revised Edition (Preliminary Version)", (1991).

[16] Calabretta, M. R., and Greisen, E. W., "Representations of celestial coordinates in FITS" (Paper II), Astronomy \& Astrophysics, 395, 1077-1122 (2002). 